\title{
Extended Indication Criteria for Endoscopic Mucosal Resection of Early Gastric Cancer with Special Reference to Lymph Node Metastasis - Examination by Multivariate Analysis -
}

\author{
KEITA NAKAHARA* ${ }^{*}$, OSAMU TSURUTA**, HIDEO TATEISHI*‡, NOBUYUKI ARIMA**, \\ JINRYO TAKEDA ${ }^{\ddagger}$, ATSUSHI TOYONAGA* $*$ AND MICHIO SATA* $*$ \\ Departments of Medicine*, Pathology**, Surgery* and Division of Gastroenterology", \\ Kurume University School of Medicine, Kurume 830-0011, Japan
}

\begin{abstract}
Summary: The purpose of this study was to clarify extended indication criteria of endoscopic mucosal resection (EMR) for early gastric cancer (EGC) by analyzing the independent risk factors involved in lymph node metastasis (LNM). Subjects were 422 patients who underwent gastrectomy with lymph node dissection for EGC at the Kurume University Hospital from 1994 to 2001. The EGCs were mucosal cancers (M) in 252 cases and submucosal cancers (SM) in 170 cases. Twelve clinico-pathological factors were assessed for their possible association with LNM. On univariate analysis, EGC with LNM showed the following characteristics: size; $3.1 \mathrm{~cm}$ or more, ulceration; present, heterogeneity; present, differentiation; poor, lymphatic vascular invasion; present, and invasion depth; SM2 (cancer penetration of submucosal layer, $0.5 \mathrm{~mm}$ or more from the muscularis mucosa). On multivariate analysis, the following four factors were identified as independent risk factors; invasion depth: Odds Ratio (OR) 10.9, lymphatic vascular invasion: OR 10.6, size: OR 3.2, and ulceration: OR 3.2. The incidence of LNM was $0 \%$ (0/141) (95\% confidence interval, $0-2.6 \%)$ when these risk factors met the following four conditions: invasion depth; M or SM1 (cancer penetration of submucosal layer, less than $0.5 \mathrm{~mm}$ ), lymphatic vascular invasion; absent, size; $3.0 \mathrm{~cm}$ or less, and ulceration; absent. It is concluded that EMR is a suitable radical treatment for EGC, and that the indication criteria for EMR can be extended depending on the results of the histological evaluation of the en bloc/total resected specimen concerning the above four factors for LNM.
\end{abstract}

Key words early gastric cancer, lymph node metastasis, endoscopic mucosal resection, multivariate analysis

\section{INTRODUCTION}

Recently, there has been much discussion of whether the indications of endoscopic mucosal resection (EMR) for early gastric cancer (EGC) should be extended with respect to improving quality of life [19]. EGC is defined as gastric cancer in which tumor invasion is confined to the mucosa and submucosa, irrespective of the presence of regional lymph node metastasis (LNM) [10]. For EMR to be considered an acceptable treatment for EGC, it must produce results similar to those obtained by surgery with respect to radicality, safety, and technique. Briefly, it will be necessary to demonstrate, by sufficient histological evaluation after treatment, that the risk of LNM is extremely low. In addition, it is essential that an en bloc/total resected specimen be collected, regardless of the location or size of the lesion [11-

Received for publication November 28, 2003

Correspondence to: Dr. Keita Nakahara, Second Department of Internal Medicine, Kurume University School of Medicine, 67 Asahi-machi, Kurume 830-0011, Japan.

Abbreviations: $95 \% \mathrm{Cl}, 95 \%$ confidence interval; EGC, early gastric cancer; EMR, endoscopic mucosal resection; LNM, lymph node metastasis; M, mucosal cancer; OR, Odds Ratio; SM, submucosal cancer; SM1, cancer penetration of submucosal layer, less than $0.5 \mathrm{~mm}$; SM2, cancer penetration of submucosal layer, $0.5 \mathrm{~mm}$ or more from muscularis mucosa. 
13].

Previously, the size and location of EGCs in which en bloc/total resection was possible was limited due to technical issues with EMR $[1,14,16]$. Therefore, indication criteria for EMR were more strict in the past [1]. Currently, the accepted indications for EMR in Japan are well -differentiated mucosal lesions less than $2 \mathrm{~cm}$ in size without ulceration. However, the recent development of new endoscopic devices, such as the insulated-tip electrosurgical knife (IT knife) [11-14] and the Hooking knife [15], has resolved many of these technical issues.

In the present study we investigated the presence or absence of LNM, which is the most important factor related to extending the indication criteria of EMR in patients with EGC, clarified the clinicopathological risk factors for LNM, and examined the conditions under which EMR can be selected as a suitable radical treatment for EGC.

\section{PATIENTS AND METHODS}

\section{Patients}

The study included 422 patients with EGCs, consisting of 252 mucosal and 170 submucosal lesions, who underwent surgery including lymph node dissection from 1994 to 2001, and whose stomach Xray/Endoscopic and macroscopic/histological findings could be adequately examined. Patients who underwent local/segmental resection, laparoscopic surgery and EMR without lymph node dissection were excluded.

\section{Statistical analysis}

All analysis was done with the Stat View software package for Macintosh (version 5.0). The chi square test, Fisher's direct test, the Mann-Whitney U test and Logistic regression analysis were performed. $\mathrm{P}<0.05$ was regarded as significant (paired test).

\section{Examination of EGC with LNM}

Univariate analysis was performed to compare the following 12 clinico-pathological factors between 30 lesions with and 392 lesions without LNM. Clinico-pathological factors were classified into the following categories in accordance with the Japanese Classification of Gastric Carcinoma [10].

1) Age: year (Mean $\pm \mathrm{SD})$.

2) Gender: 2 categories; male and female.

3) Location: 3 categories; U: upper third of stomach, M: middle third of stomach, and L: lower third of stomach.

4) Regional wall: 4 categories; the lesser curvature, greater curvature, anterior wall, and posterior wall.

5) Macroscopic type: 3 categories; depressed type, protruded type, and mixed type.

6) Size: 2 categories; $3.0 \mathrm{~cm}$ or less and $3.1 \mathrm{~cm}$ or more.

7) Histological type: 2 categories; undifferentiated type (poorly differentiated adenocarcinoma, signet-ring cell carcinoma) and differentiated type (tubular adenocarcinoma, papillary adenocarcinoma).

8) Heterogeneity: 2 categories; absent and present. When the above 2 histological types were mixed, lesions were defined as "present".

9) Differentiation: 3 categories; well, moderate, and poor. Differentiation was defined as representing not the quantitatively predominant histological type but the most poorly differentiated areas.

10) Ulceration: 2 categories; absent and present. Lesions in which macroscopic and histological findings showed fold convergence or submucosal fibrosis were defined as "present".

11) Lymphatic vascular invasion: 2 categories; absent and present.

12) Invasion depth: 3 categories; M, SM1 and SM2. Among lesions infiltrating the submucosal layer, those in which the infiltration distance from the muscularis mucosa was less than $0.5 \mathrm{~mm}$ were evaluated as SM1, while those in which the distance was $0.5 \mathrm{~mm}$ or more were evaluated as SM2.

\section{Examination of risk factors for LNM}

Multivariate analysis (logistic regression analysis) was performed using the presence or absence of LNM as an objective variable and the above factors selected by univariate analysis as explanatory variables. To select variables that may be risk factors for LNM, the variable-increasing method using the likelihood ratio test was used. Explanatory variables with a correlation coefficient of 0.9 or more or with multiple commensal linearity were excluded. The odds ratio (OR) in each factor and $95 \%$ confidence interval $(95 \% \mathrm{CI})$ were calculated.

\section{Examination of indication criteria for EMR}

Stratified analysis was performed based on risk factors for LNM selected by multivariate analysis. We investigated conditions showing the lowest risk 
of LNM.

\section{RESULTS}

\section{Examination of EGC with $L N M$}

EGC with LNM showed the following characteristics in comparison to EGC without LNM; size: 3.1 $\mathrm{cm}$ or more, ulceration: present, heterogeneity: present, differentiation: poor, lymphatic vascular inva- sion: present, and invasion depth: SM2. There were no significant differences in age, gender, location, regional wall, macroscopic type, or histological type (Table 1).

\section{Examination of risk factors for $L N M$}

The following four factors were identified as independent risk factors for LNM among the above six factors, namely invasion depth: $\mathrm{P}=0.0311$, OR10.85, lymphatic vascular invasion: $\mathrm{P}<0.0001$,

TABLE 1.

Characteristics of EGC with LMN by univariate analysis

\begin{tabular}{|c|c|c|c|c|}
\hline \multicolumn{2}{|c|}{ Clinico-pathological factors } & \multirow{2}{*}{$\begin{array}{l}\text { LMN: absent } \\
64.32 \pm 10.81\end{array}$} & \multirow{2}{*}{$\begin{array}{l}\text { LMN: present } \\
66.47 \pm 8.31\end{array}$} & \multirow{2}{*}{$\begin{array}{r}P \text {-value } \\
P=0.2605\end{array}$} \\
\hline Age & Mean \pm SD & & & \\
\hline \multirow[t]{2}{*}{ Gender } & Male & $269(68.6 \%)$ & $20(66.7 \%)$ & $P=0.8241$ \\
\hline & Female & $123(31.3 \%)$ & $10(33.3 \%)$ & \\
\hline \multirow[t]{3}{*}{ Location } & $\mathrm{U}$ & $76(19.4 \%)$ & $5(16.7 \%)$ & $P=0.4276$ \\
\hline & M & $101(25.8 \%)$ & $11(36.7 \%)$ & \\
\hline & $\mathrm{L}$ & $215(54.8 \%)$ & $14(46.7 \%)$ & \\
\hline \multirow[t]{4}{*}{ Regional wall } & Posterior wall & $95(24.2 \%)$ & $7(23.3 \%)$ & $P=0.3770$ \\
\hline & Anterior wall & $83(21.2 \%)$ & $8(26.7 \%)$ & \\
\hline & Lesser curvature & $157(40.1 \%)$ & $8(26.7 \%)$ & \\
\hline & Greater curvature & $57(14.5 \%)$ & $7(23.3 \%)$ & \\
\hline \multirow[t]{3}{*}{ Macroscopic type } & Depressed type & $273(69.6 \%)$ & $17(56.7 \%)$ & $P=0.3154$ \\
\hline & Protruded type & $51(13.0 \%)$ & $5(16.7 \%)$ & \\
\hline & Mixed type & $68(17.3 \%)$ & $8(26.7 \%)$ & \\
\hline \multirow[t]{2}{*}{ Size } & $3.0 \mathrm{~cm}$ or less & $263(67.1 \%)$ & $8(26.7 \%)$ & $P<0.0001$ \\
\hline & $3.1 \mathrm{~cm}$ or more & $129(32.9 \%)$ & $22(73.3 \%)$ & \\
\hline \multirow[t]{2}{*}{ Histological type } & Undifferentiated type & $142(36.2 \%)$ & $8(26.7 \%)$ & $P=0.2918$ \\
\hline & Differentiated type & $250(63.8 \%)$ & $22(73.3 \%)$ & \\
\hline \multirow[t]{2}{*}{ Heterogeneity } & absent & $311(79.3 \%)$ & $14(46.7 \%)$ & $P<0.0001$ \\
\hline & present & $81(20.7 \%)$ & $16(53.3 \%)$ & \\
\hline \multirow[t]{3}{*}{ Differentiation } & well & $153(39.0 \%)$ & $1(3.3 \%)$ & $P=0.0004$ \\
\hline & moderate & $58(14.8 \%)$ & $8(26.7 \%)$ & \\
\hline & poor & $181(46.2 \%)$ & $21(70.0 \%)$ & \\
\hline \multirow[t]{2}{*}{ Ulceration } & absent & $220(56.1 \%)$ & $5(16.7 \%)$ & $P<0.0001$ \\
\hline & present & $172(43.9 \%)$ & $25(83.3 \%)$ & \\
\hline \multirow{3}{*}{$\begin{array}{l}\text { Lymphatic vascular } \\
\text { invasion }\end{array}$} & absent & $349(89.0 \%)$ & $6(20.0 \%)$ & $P<0.0001$ \\
\hline & present & $43(11.0 \%)$ & $24(80.0 \%)$ & \\
\hline & M & $251(64.0 \%)$ & $1(3.3 \%)$ & $P<0.0001$ \\
\hline \multirow[t]{2}{*}{ Invasion depth } & SM1 & $42(10.7 \%)$ & $3(10.0 \%)$ & \\
\hline & SM2 & $99(25.3 \%)$ & $26(86.7 \%)$ & \\
\hline
\end{tabular}

TABLE 2.

Risk factors for LNM in EGC by multivariate analysis

\begin{tabular}{lllcc}
\hline \multicolumn{2}{c}{ Clinico-pathological factors } & $P$-value & Odds ratio & 95\%CI \\
\hline Invasion Depth & SM1 vs M & $P=0.1580$ & 5.66 & $0.51-62.7$ \\
& SM2 vs M & $P=0.0311$ & 10.85 & $1.24-94.8$ \\
Lymphatic vascular & present vs absent & $P<0.0001$ & 10.56 & $3.64-30.6$ \\
invasion & & & 3.22 & $1.23-8.42$ \\
Size & $3.1 \mathrm{~cm}-$ vs $-3.0 \mathrm{~cm}$ & $P=0.0175$ & 3.21 & $1.06-9.73$ \\
Ulceration & present vs absent & $P=0.0394$ & \\
\hline
\end{tabular}


TABLE 3.

Stratified analysis of 4 risk factors for $L M N$ in EGC

\begin{tabular}{|c|c|c|c|c|c|c|c|c|c|}
\hline \multirow{5}{*}{$\begin{array}{l}\text { Risk } \\
\text { Factors }\end{array}$} & & \multicolumn{4}{|c|}{ M or SM1 } & \multicolumn{4}{|c|}{ SM2 } \\
\hline & & \multirow{2}{*}{\multicolumn{4}{|c|}{$\begin{array}{l}\text { Lymphatic vascular invasion } \\
\text { absent }\end{array}$}} & \multicolumn{4}{|c|}{ Lymphatic vascular invasion } \\
\hline & & & & & & & & pre & \\
\hline & & \multicolumn{2}{|c|}{ LNM } & \multicolumn{2}{|c|}{ LNM } & \multicolumn{2}{|c|}{ LNM } & \multicolumn{2}{|c|}{$\mathrm{LNm}$} \\
\hline & & absent & present & absent & present & absent & present & absent & present \\
\hline \multirow{2}{*}{$-3.0 \mathrm{~cm}$} & Ulceration & 141 & 0 & 3 & 0 & 19 & 0 & 8 & 0 \\
\hline & present & 61 & 1 & 4 & 0 & 18 & 1 & 9 & 6 \\
\hline \multirow{2}{*}{$3.1 \mathrm{~cm}-$} & Ulceration & 34 & 0 & 2 & 1 & 8 & 1 & 5 & 3 \\
\hline & present & 47 & 1 & 1 & 1 & 21 & 2 & 11 & 13 \\
\hline Total & & 283 & 2 & 10 & 2 & 66 & 4 & 33 & 22 \\
\hline
\end{tabular}

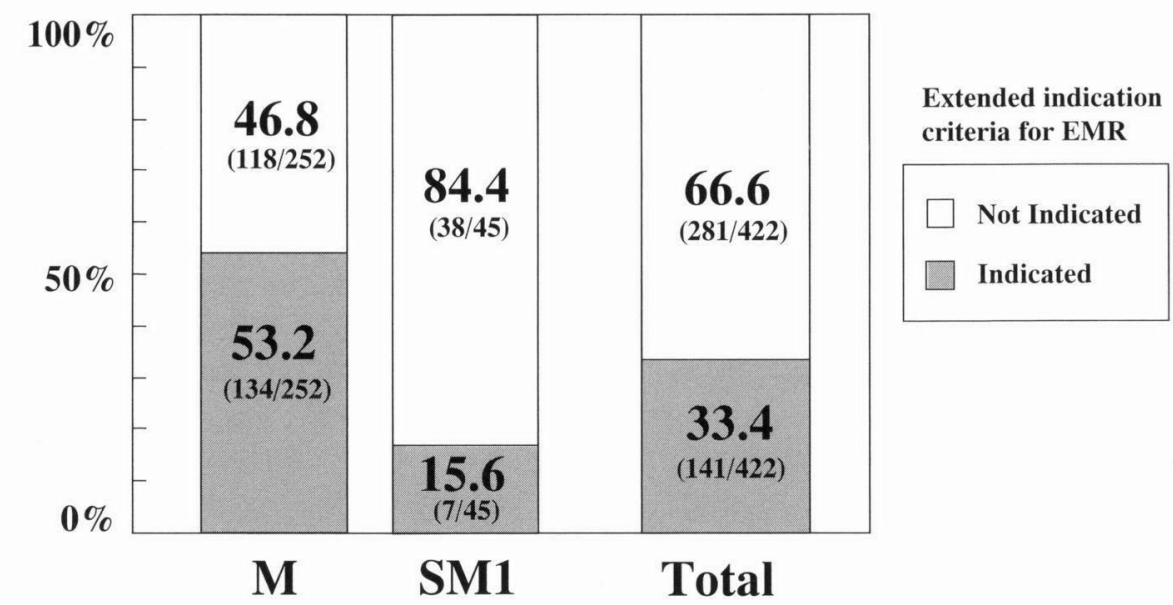

Fig. 1. The percentages of cases in which EMR is indicated among the EGC patients who underwent surgery.

TABLE 4.

Extended indication criteria for EMR of EGC

$\begin{array}{ll}\text { Invasion depth } & : \text { M or SM1 } \\ \text { Lymphatic vascular invasion } & : \text { absent } \\ \text { Size } & : 3.0 \mathrm{~cm} \text { or less } \\ \text { Ulceration } & : \text { absent } \\ \text { Lymph node metastasis : } 0 \%(0 / 141), 95 \% \text { CI: } 0-2.6 \%\end{array}$

OR10.56, size: $\mathrm{P}=0.0175$, OR3.22, and ulceration: $\mathrm{P}=0.0394$, OR3.21 (Table 2).

Concerning the overall adaptability of multivariate analysis, the likelihood ratio was $\mathrm{P}<0.0001$. When likelihood ratio tests for individual variables were conducted, the following values were obtained; invasion depth: $\mathrm{P}=0.0294$, lymphatic vascular invasion: $\mathrm{P}<0.0001$, size: $\mathrm{P}=0.0139$, and ulceration: $\mathrm{P}=0.0284$. These variables were essential for analysis.

\section{Examination of the indication criteria for EMR}

The four conditions with the lowest risk of LNM were: invasion depth; M or SM1, lymphatic vascular invasion; absent, size; $3.0 \mathrm{~cm}$ or less, and ulceration; absent. When lesions met these conditions the incidence of LNM was $0 \%(0 / 141)(95 \% \mathrm{CI}, 0-2.6 \%)$ (Tables 3, 4).

Among EGC lesions surgically resected in our hospital, 53.2\% met the four conditions (134/252, 95\% CI: $46.8-59.5 \%)$ in the case of $\mathrm{M}$ and $15.6 \%$ (7/45, 95\% CI: $6.4-29.5 \%)$ for SM1. Overall, the rate was 33.4\% (141/422, 95\%CI: 28.9-38.1\%) (Fig. 1). 


\section{DISCUSSION}

Many previous retrospective studies reported on clinico-pathological conditions regarding LNM in EGC and extended criteria for EMR [1-9]. However, the statistical limitations of univariate analysis in these retrospective studies include randomization, selection bias, survey omission, and confounding factors. Therefore, it has been pointed out that study design and analytic evaluation should be performed more carefully than in prospective studies [17]. Multivariate analysis is has recently been used as a more appropriate statistical procedure. However, previous studies have mainly analyzed LNM with respect to the histological type [18], macroscopic type [19], size [20], or have investigated submucosal invasive cancer alone [21-23].

In the present study, we investigated the risk factors for LNM using multivariate analysis in patients with EGC (mucosal and submucosal cancers) who underwent surgery. Initially, univariate analysis revealed that EGC with LNM showed a larger lesional diameter, a higher incidence of ulceration, more marked heterogeneity, poorly differentiated areas, and deeper infiltration (Table 1). Considering these characteristics, multivariate analysis was performed to adjust confounding factors among individual clinico-pathological factors. As a result, the following four factors were extracted as the independent risk factors for LNM: invasion depth; OR10.85, lymphatic vascular invasion; OR10.56, size; OR3.22, and ulceration; OR3.21. It was shown that the risk of LNM increased with the odds ratio (Table 2).

In addition, we investigated the conditions for adoption of EMR. In resected EGC lesions the incidence of LNM was $0 \%$ (0/141) (95\%CI: $0-2.6 \%$ ) (Tables 3,4) when the four independent risk factors identified above met the following conditions: invasion depth; M or SM1, lymphatic vascular invasion; absent, size; $3.0 \mathrm{~cm}$ or less, and ulceration; absent. The results of this study were similar to those reported by several other investigators with respect to these four factors [1-9,18-23]. However, multivariate analysis showed that the above four factors independently and markedly influenced LNM, and that the histological type was not a risk factor for LNM. There was no significant difference in invasion depth between SM1 and M cancers among EGCs. This is an important finding with regard to extended adoption of EMR.
With respect to size and ulceration, the possibility of SM invasion is higher and ulceration develops more readily in larger lesions. In addition, from the technical perspective of en bloc/total resection, a condition of $3 \mathrm{~cm}$ or less may be considered safe.

As for lymphatic vascular invasion, this may be the most important direct route to the lymph nodes. However, it is impossible to evaluate the presence or absence of lymphatic vascular invasion preoperatively, therefore postoperative histological evaluation is essential.

With respect to histological type, EMR has previously been indicated only for differentiated cancers [1]. However, this study suggested that EMR is applicable for lesions meeting the above four conditions regardless of whether they were differentiated or undifferentiated. In clinical practice, non-serial infiltration sometimes makes it difficult to identify the extent of infiltration and to evaluate the stump in undifferentiated type, and this is a diagnostic issue [7]. Currently, careful local treatment is indicated, and sufficient investigation of this issue should be performed in the future.

As described in the Introduction, to more accurately evaluate the status of these four risk factors, an EMR procedure must be employed, in which an en bloc/total resected specimen can be obtained, there by facilitating evaluation similar to that of surgery [3,11-15]. When resection is fragmented, the bruised margin makes it difficult to evaluate the stump, and the degree of radical treatment cannot be adequately evaluated. This issue is particularly important in lesions for which local treatment is indicated. When en bloc/total resection is technically impossible, indications of EMR should not be readily extended.

In approximately $30 \%$ (Fig. 1) of patients with EGC who underwent surgery in our hospital, it was suggested that EMR was a suitable radical treatment. However, a large-scale prospective study should be carried out to determine whether the extended criteria for EMR in this study (Table 4) can be applied in clinical practice. Furthermore, not only treatment techniques but also the accuracy of preoperative diagnosis must be further improved in order to promote this treatment in clinical practice.

In conclusion, EMR is a suitable radical treatment for EGC. Based on the extended criteria identified in this study, it is expected that EMR can replace surgery in more EGC cases that had previously been indicated. 


\section{REFERENCES}

1. Japanese Gastric Cancer Association. Guidelines of treatment for gastric cancer, Kanehara, Tokyo, 2001. (in Japanese)

2. Gotoda T, Sasako M, Shimoda T, Ono H, and Katai $\mathrm{H}$ et al. An evaluation of the necessity of gastrecomy with lymph node dissection for patients with submucosal invasive gastric cancer. Br J Surg 2001; 88:444-449.

3. Gotoda T, Yanagisawa A, Sasako M, Ono H, Nakanishi $Y$ et al. Incidence of lymph node metastasis from early gastric cancer, estimation with a large number of cases at two centers. Gastric Cancer 2000; 3:219-225.

4. Yasuda K, Shiraishi N, Suematsu T, Yamaguchi K, Adachi $\mathrm{Y}$ et al. Rate of detection of lymph node metastasis is correlated with the depth of submucosal invasion in early stage gastric carcinoma. Cancer 1999; 85:21192123.

5. Yamano T, Shirao K, Ono H, Kondo H, Saito D et al. Risk factors for lymph node metastasis from intramucosal gastric carcinoma. Cancer 1996; 77:602-606.

6. Tsuji N, Ishiguro S, Kasugai T, Suzuki N, Miwa H et al. Risk factor for lymph node metastasis of submucosal invasive carcinoma. Stomach and Intestine 1997; 32:4147. (in Japanese)

7. Nakao K, Yanagisawa A, Utsude T, Kubo K, and Kato $Y$. Limits of indication of endoscopic mucosal resection (EMR) for treatment of early gastric carcinoma of undifferentiated type-from a pathological viewpoint. Stomach and Intestine 1995; 30:1289-1294. (in Japanese)

8. Tsuchiya A, Kikuchi Y, Ando Y, Yoshida T, and Abe R. Lymph node metastasis in gastric cancer invading the submucosal layer. Eur J Surg Oncol 1995; 21:248-250.

9. Iwashita M, Furukawa K, Yamada Y, Yao T, Arima S et al. Pathomorpholigic characteristics of early gastric carcinoma with nodal involvement. Endoscopia digestiva 1995; 7:697-707. (in Japanese)

10. Japanese Gastric Cancer Association. Japanese classification of gastric carcinoma. Gastric cancer, 2nd English edn, Kanehara, Tokyo, pp10-24, 1998. (in Japanese)

11. Hosokawa K, and Yoshida S. Recent advances in endoscopic mucosal resection for early gastric cancer. Jpn $\mathbf{J}$ Cancer Chemother 1998; 25:476-483.

12. Ohkura M, Hosokawa N, Boku N, Ohtsu H, Tajiri S et al. New Endoscopic treatment technique for intramucosal gastric tumors using an insulated-tip diathermic knife. Endoscopy 2001; 33:221-226.
13. Ono H, Kondo H, Gotoda T, Shirao K, Yamaguchi H et al. Endoscopic mucosal resection for treatment of early gastric cancer. Gut 2001; 48:225-229.

14. Miyamato S, Muto M, Hanamoto Y, Boku N, Ohtsu A et al. A new technique for endoscopic mucosal resection with an insulation-tipped electro surgical knife improves the completeness of resection of intramucosal gastric neoplasms. Gastrointest Endosc 2002; 50:576-581.

15. Oyama T, Kikuchi Y, Shimaya S, Tomori A, Hotta K et al. Endoscopic mucosal resection using a Hooking knife (Hooking EMR). Stomach and Intestine 2002; 37:11551161. (in Japanese)

16. Tada M, Murakami A, Karita M, Yanai H, and Okita K. Endoscopic resection of early gastric cancer. Endoscopy 1993; 25:445-450.

17. The Japanese society of Gastroenterology. Questions \& Answers in MEDICAL BIOSTATISTICS, 1st edn, Kanehara, Tokyo, 1987. (in Japanese)

18. Mita T, and Shimoda T. Risk factors for lymph node metastasis in submucosal invasive differentiated type gastric carcinoma, clinical significance of histological heterogeneity. J Gastroenterol 2001; 36:661-668.

19. Abe N, Watanabe T, Suzuki K, Machida H, Toda H et al. Risk factors predictive of lymph node metastasis in depressed early gastric cancer. Am J Surg 2002; 183:168-172.

20. Yokota T, Saito T, Teshima S, Yamada Y, Iwamoto K et al. Probability of lymph node metastasis in small gastric cancer tumor, is it an indication for limited surgery? Int Surg 2001; 86:206-209.

21. Nishida T, Tanaka S, Haruma K, Ohtsu N, Tsuda T et al. Histologic grade and cellular proliferation at the deepest invasive portion correlate with the high malignancy of submucosal invasive gastric carcinoma. Oncology 1995; 52:340-346.

22. Kajiyama Y, Tsurumaru A, Udagawa H, Tsutsumi K, Kinoshita $\mathrm{Y}$ et al. Possibility of reduction in lymph node dissection in gastric submucosal cancer-in view of prediction of lymph node metastasis by logistic regression analysis, and indication for laparoscopic resection. Stomach and Intestine 1997; 32:63-69. (in Japanese)

23. Yasuda M, Niki M, Torisu R, Bandou G, Hayashi T et al. Indications of endoscopic mucosal resection (EMR) for submucosal invasive gastric cancer with special reference to histopathologic findings-research using the logistic regression analysis method Gastroenterol Endosc 2003; 45:233-240. (in Japanese) 